\title{
An Ion Current Based Peak-Finding Algorithm for Pressure Peak Position Estimation
}

\author{
Magnus Hellring, Ulf Holmberg \\ Halmstad University
}

Copyright $(1998$ Society of Automotive Engineers, Inc.

\begin{abstract}
In this paper a novel ion current based estimation scheme for the in-cylinder pressure peak position (PPP) is proposed. A reliable estimate is constructed by appropriate signal processing based on local curvatures of the post flame phase of the ion current. The peakfinding algorithm is simple and easy to implement in an engine control unit for feedback control of the combustion phasing. Results on real data, sampled onboard a commercial car are presented. Further, the performance of the algorithm is compared to two state of the art algorithms for PPP estimation from the ion current. The comparison shows that the algorithm presented in this paper outperforms its competitors ${ }^{1}$.
\end{abstract}

\section{INTRODUCTION}

Production systems of today use feedforward control algorithms to compromise between fuel economy, engine performance and emission levels. These are implemented as static look up tables, based on sensing of parameters such as: RPM, manifold air pressure, throttle angle, coolant temperature and atmospheric pressure. This control strategy is sensitive to e.g. engine diversities, engine aging, fuel additives and environmental disturbances like humidity. Thus, optimal performance can not be guaranteed.

Regarding fuel consumption and efficiency of the spark ignited engine; a close relationship with the in-cylinder pressure peak position (PPP) has earlier been reported [1]. Moreover, feedback control using an intrusive pressure sensor to assess PPP as feedback variable has previously been successfully demonstrated [1, 2]. However, the drawbacks of in-cylinder pressure sensing, i.e. cavity forming due to the intrusive measurement technique, a significant baseline drift and high cost, are reasons why pressure sensors are not used in mass production.

\footnotetext{
${ }^{1}$ To the authors' knowledge, there is only two ion current based algorithms described in the literature.
}

The ionization of the combustion gas versus the crank angle, referred to as the ion current signal, is rich on information about the combustion. In particular, the end phase of the signal, corresponding to thermal ionization, can be related to the in-cylinder pressure. Various schemes have therefore been suggested for estimation of the in-cylinder pressure peak position (PPP) from the ion current signal $[3,4,5]$. These are either based on curve-fitting or pattern-matching techniques. Although yielding good results, they all suffer from signal alterations related to the ion current measuring technique. Especially low engine load deteriorates ion current signal quality in terms of correspondence to the in-cylinder pressure.

The approach herein estimates the PPP from the ion current using a simple curvefitting technique. The algorithm is fast and requires a small amount of storage and computation.

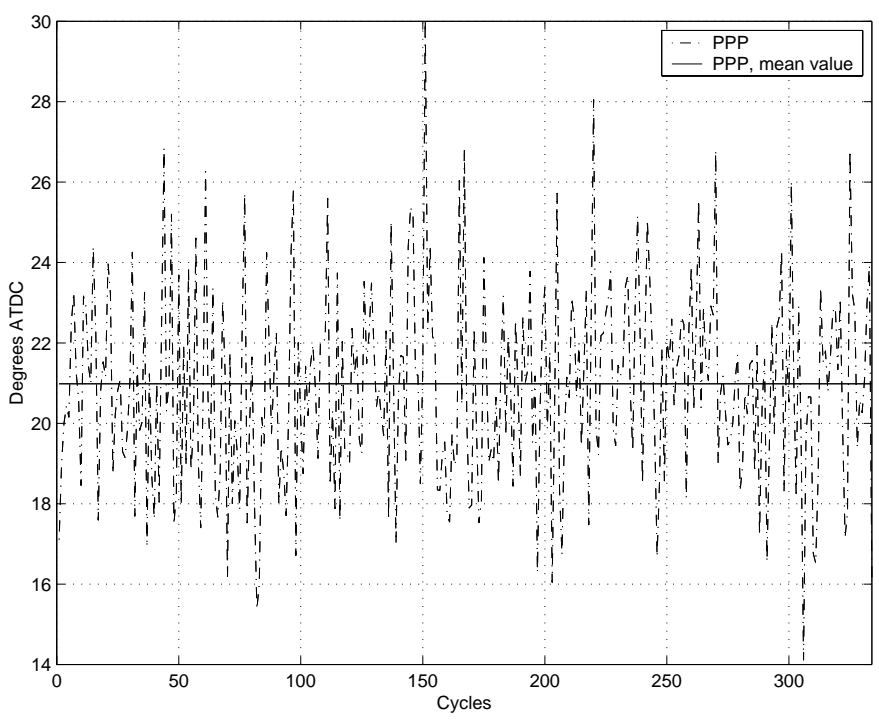

Figure 1. Cycle to cycle variations in PPP (Dash-dotted line). PPP is calculated from the pressure trace, which has been sampled in a laboratory environment under constant external conditions. The engine speed was 2700 RPM and the load was $40 \%$ of MBT. The mean PPP (Solid line) is 20.9 degrees ATDC and the standard deviation is 2.4 degrees. 


\section{PRESSURE PEAK POSITION}

Optimal spark advance in terms of efficiency maintains an almost constant combustion phasing no matter what external disturbances that are present [2]. This can be accomplished using feedback control, where the result of the spark setting is continuously measured [1]. The combustion phasing can be described by e.g. PPP or MFB50\%. In this paper, PPP is chosen. Moreover, the purpose with the developed ion current based PPP estimator is to replace the intrusive pressure sensor.

The intrinsic noise in PPP is quite high resulting in a standard deviation of approximately 2.5 crank angle degrees during both constant operation and constant external conditions, Fig. 1. This is due to cycle to cycle variations of the combustion engine and ambiguities related to the sometimes flat peak of the pressure trace. Nevertheless, spark advance control of PPP is quite robust and cycle to cycle variations result in less than $0.4 \%$ loss in net indicated work [3]. Accordingly, an estimator for PPP might disregard cycle to cycle variations without deterioration of the control scheme.

\section{ION CURRENT}

The ion current is measured in the combustion chamber, using the sparkplug as sensor. The sensing technique is to apply a DC bias to the spark plug when it is not used for ignition and measure the current that flows through the circuit. This type of ion current measuring is already used for e.g. knock - and misfire detection in production cars of today.

The ion current can be divided into three phases: the ignition phase, were the ion current makes large peaks and ringing of the electronic measurement device, the flame-front phase, which typically displays one or several large maxima, and the post flame phase, where (sometimes) a maximum corresponding to the position of the pressure peak is displayed. These phases can be difficult to discern when examining the ion current from a single cycle and are therefore often illustrated using ion currents that have been sampled during constant external conditions and then averaged over several consecutive cycles, Fig. 2. In contrast to the "ideal" ion current signal in Fig. 2, the cycle-to-cycle ion current, shown in Fig. 3, varies significantly between cycles. It is unlikely that one encounters an example like the one illustrated in Fig. 2.

Moreover, when the ion current has been obtained outside the laboratory, i.e. onboard an "off the shelf" commercial car, additional external disturbances like temperature and humidity appear.

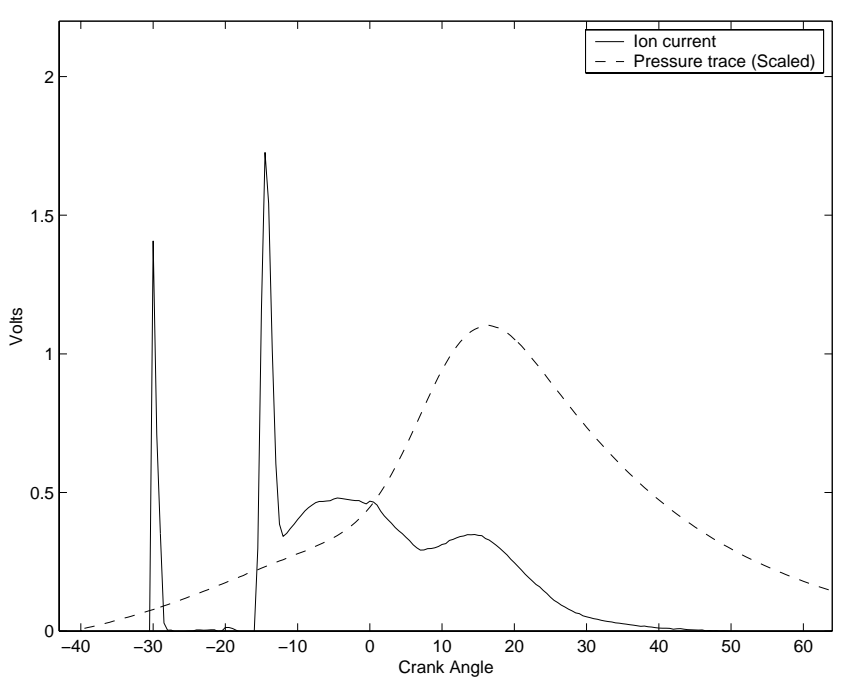

Figure 2. An "ideal" ion current signature (solid line): The signal has been sampled in a laboratory environment under constant external conditions. The engine speed was 1500 RPM and the load was $70 \%$ of MBT. The pressure trace is shown for comparison (dashed line). The plotted signals are the result of averaging over 100 consecutive cycles

A simple theoretical model, assuming e.g. adiabatic conditions, says that the ion current peak in the post flame phase (the rightmost peak in Fig. 2) should coincide with the pressure peak under high load conditions [6]. Empirical studies [7], under steady state conditions in a dynamometer, indicate that this is approximately true but that the model has difficulties describing low load phenomena, even if data is collected under ideal external conditions and then averaged. This complication is due to the fact that the post flame peak essentially vanishes if the load is less than $20 \% \mathrm{MBT}^{2}$ (see Fig. 3). It is worth mentioning, in this context, that normal driving on the highway is close to the low load situation.

In order for an ion current based PPP estimator to be commercially viable, it must improve performance when used in feedforward control. Therefore, the PPP estimates must not be biased or easily corrupted by ion current signal alterations. Thus, the solution proposed here utilizes bounds on the ion current signal shape when searching for PPP. 


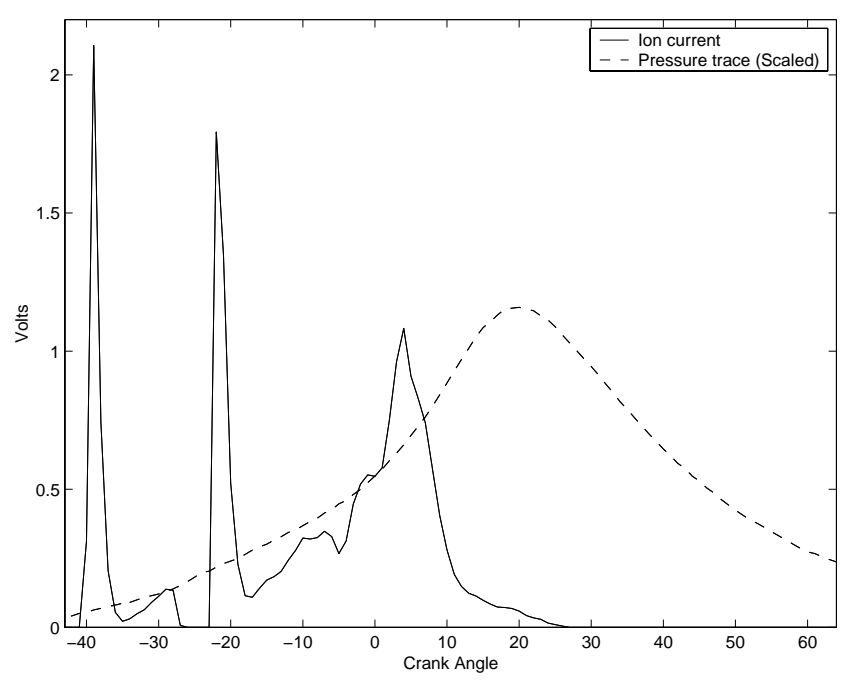

Figure 3. An ion current signal sampled during a single cycle on board the SAAB car while driving on the highway (solid line). The engine speed was 1500 RPM and the load was approximately $25 \%$ of MBT. There are several wide peaks in the signal and the post flame phase is not visible. The pressure trace is shown for comparison (dashed line).

\section{PEAK-FINDING ALGORITHM}

The approach in this paper operates on single cycle ion currents and relies on the assumption of high correlation between the post flame phase peak of the ion current and the PPP. Thus, it is assumed that the flame front phase is detectable using simple signal processing.

\section{THE IDEA}

The idea is to successively fit a second order polynomial to locally describe the ion current signal as a function of crank angle. From the local model, the second derivative is calculated which gives information about how the slope is changing. If the second derivative is negative (positive) the model has a maximum (minimum). By scanning from the largest to smaller crank angles and comparing the second derivatives of the fitted local models, the local maximum of the ion current that corresponds to PPP can be found. Since there are other local maxima from the flame front phase the scanning of local models should be able to detect the end of the flame front phase and stop the scanning. This is done by giving a threshold for the maximum allowed second derivative. When passing this threshold, the end of the flame front is considered found, see Fig. 4.

The remaining ion current signal corresponding to higher crank angles then belongs to the post flame phase. The local model having the smallest second derivative is then chosen and used for calculation of the PPP estimate. The PPP estimate is chosen as the crank angle where the first derivative is zero. This now corresponds to the sharpest maximum in the post flame phase, see Fig. 4a. a)

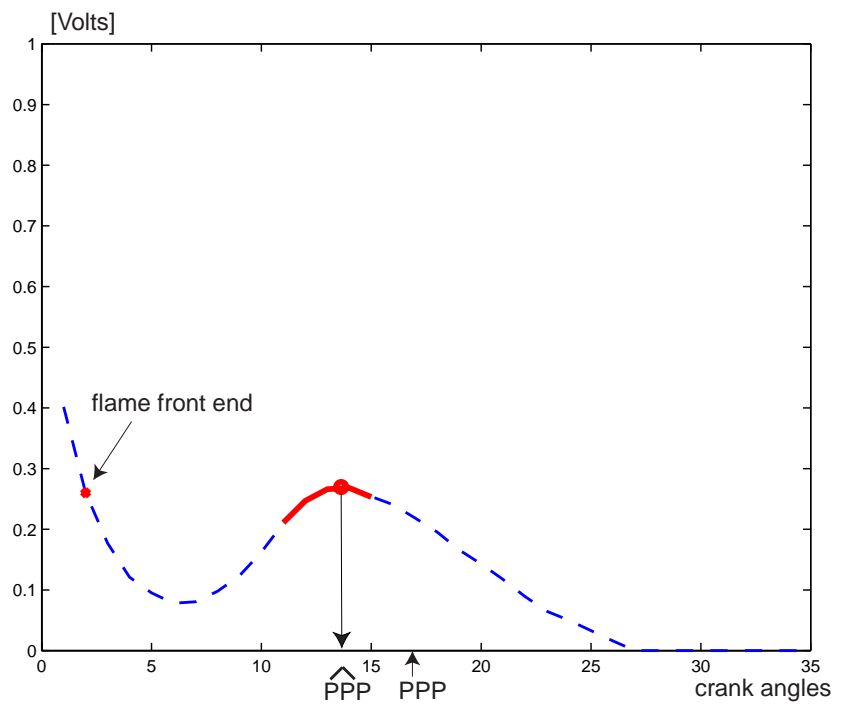

b)

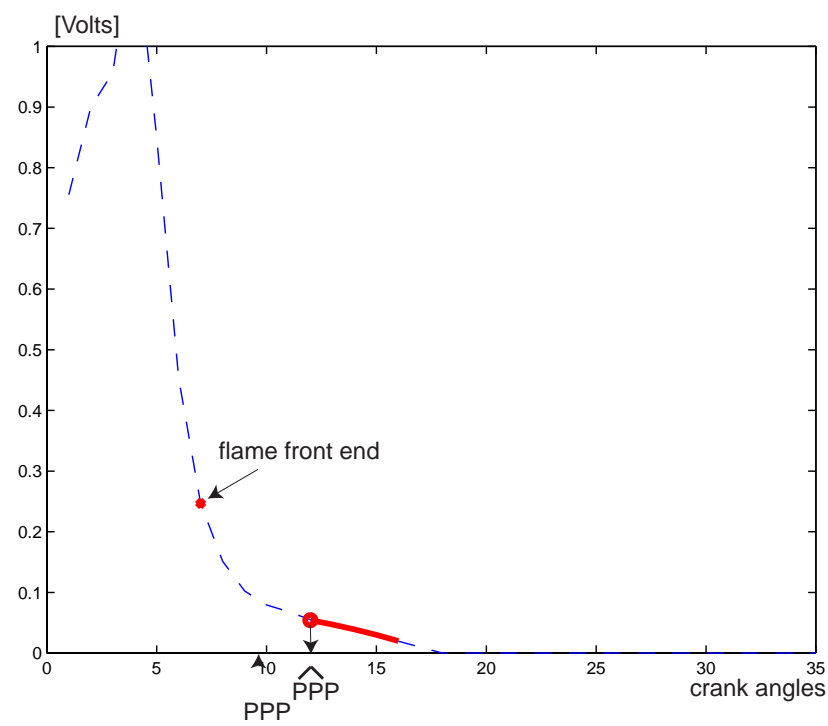

c)

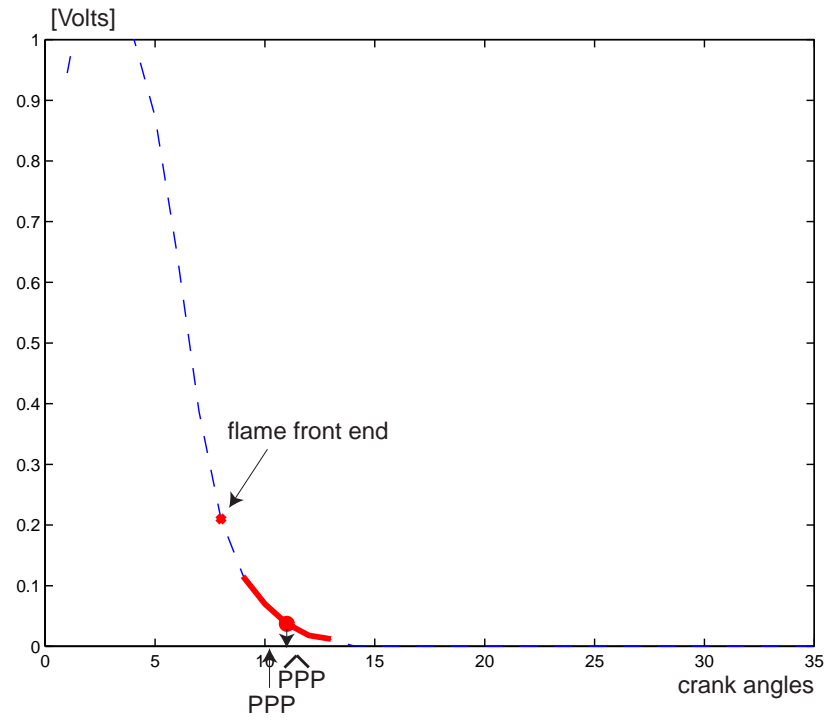

Figure 4. Estimations using the peak-finding algorithm. PPP corresponds to the pressure peak position obtained from the pressure trace and PPP corresponds to the estimate. a) Local maximum is detected within the sliding window. b) The local maximum is outside the sliding window. The estimate is constrained to the closest boundary (of solid curve). c) No local maximum. Mid-point (in sliding window) of smallest second derivative is taken as estimate. 
If the calculated maximum of the model is outside its range of validity, the closest point inside the sliding window (fitted range of the local model) is chosen instead, see Fig.4b. Sometimes it may happen that the smallest second derivative is positive. The local model then has no maximum. The mid-point of the sliding window is chosen in this case, see Fig.4c.

\section{THE ALGORITHM}

Let the ion current signal be $i(\theta)$ where $\theta$ is the crank angle. The local second order model is:

$$
f(\theta)=p_{0}+p_{1} \theta+p_{2} \theta^{2}
$$

where $p_{k}, \mathrm{k}=0,1,2$ are model parameters to be estimated as below. The local model should approximate the ion current signal over a sliding window(explained below), i.e. find $p_{k}$ such that $f(\theta) \approx i(\theta)$, where the sliding window is $\theta \in\left[\theta_{1}, \theta_{2}, \ldots \theta_{n}\right]$. Since there are three unknown parameters $\left(p_{k}, \mathrm{k}=0,1,2\right)$ it is necessary to have a sliding window of at least three crank angle degrees. But, in order to reduce noise effects $n=5$ is chosen. The model fit is thus made in the least squares sense.

$$
\min _{p_{0}, p_{1}, p_{2}} \sum_{j=1}^{5}\left[f\left(\theta_{j}\right)-i\left(\theta_{j}\right)\right]^{2}
$$

This is a linear least square problem with analytical solution. From the model, the second derivative is calculated as

$$
\frac{d^{2}}{d \theta^{2}} f(\theta)=2 p_{2}
$$

When $p_{2}$ is above a chosen threshold, the flame front end is considered found. The model with the smallest $p_{2}$ is then considered. For this model, the local maximum is found where the first derivative is zero, i.e.

$$
\frac{d}{d \theta} f(\theta)=0 \Rightarrow \theta^{*}=-\frac{p_{1}}{2 p_{2}}
$$

Finally, the estimate is constrained to be inside the validity range of the estimated local model.

$$
\hat{P P P}=\min \left[\max \left[\theta_{1}, \theta^{*}\right] \theta_{5}\right]
$$

The sliding window has a low pass filtering effect and thus removes noise effects. This makes the algorithm robust towards outliers not related to the pressure peak.

\section{DATA}

Data was collected on a car during normal operation on the highway. The experimental car is a production 1996 SAAB 9000, with a 2.3-liter low-pressure turbo engine (B234E MY96). Besides standard equipment, the car has been equipped with Kistler 6121 pressure sensors.

The complete data set consists of 99000 cycles obtained in cylinder 1, from which 3 batches have been selected as test data, see Fig.5, 6 and 7 .

\section{EXPERIMENT}

The peak-finding algorithm presented in this paper is evaluated on three batches of real data describing different steps in PPP. Regarding estimator quality, bias is the primary variable of interest. A feedback controller will not be able to eliminate steady state errors if the sensor signal is biased. In order to clearly display bias errors, the PPP estimates and the PPP itself are low pass filtered in the figures in this section. This will impose approximately the same signal behavior as seen by a closed loop control system as far as bias is concerned. Furthermore, the results of the peak-finding algorithm are compared to those produced by: 1.) The Gaussian fit model presented in [3]. 2.) The MLP model presented in [4]. Accordingly, the estimates of the latter algorithms are also low pass filtered.

\section{THE MLP MODEL}

Being a parameterized model estimated from data, the MLP (Multilayer Perceptron) is sensitive to signal levels and input data dimension. Hence, a suitable preprocessing of data is needed in order to improve both estimation time and model performance. Since the algorithm requires a small amount of computation, it is very fast. However, the drawback of the algorithm is the storage needed for the parameters of the model and the preprocessing stage corresponding to approximately 1 kilobyte. For further reading see [4].

\section{THE GAUSSIAN FIT ALGORITHM}

The Gaussian fit algorithm suggested by Eriksson and Nielsen is described in some detail in e.g. [3]. The key idea in the algorithm is that the ion current can be modeled by a sum of Gaussians.

The algorithm used here is based on three Gaussians in the model function. Eriksson suggests using two [3], but our experience is that three tends to work better. We used a second order Levenberg-Marquardt algorithm for the iterative search and tried 50 different initial conditions for the parameters (the result turned out to be quite sensitive to the initial conditions). The best fit of these 50 was used for the PPP estimate. The Gaussian fit algorithm requires no significant storage but the computations needed to produce good estimates are large. Thus, a real-time implementation that works not 
only during high load might be difficult to achieve. For further reading see [3].

\section{RESULTS}

The first batch, Fig. 5, is a step in PPP from approximately 20 degrees ATDC to 10 degrees ATDC. The true PPP is obtained from the pressure trace and then filtered.

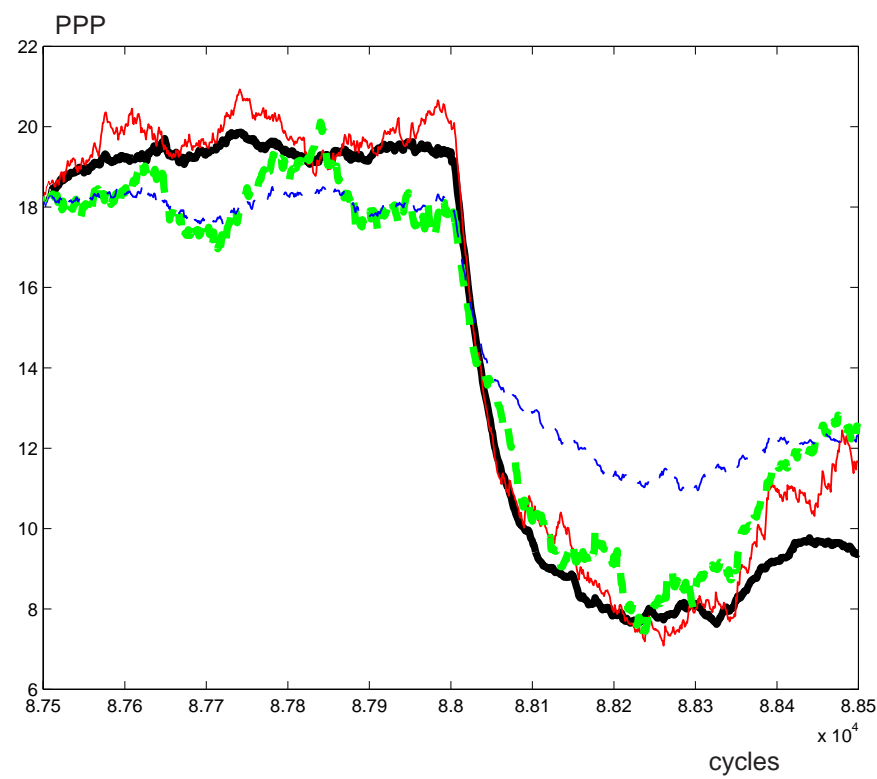

Figure 5. Step in low pass filtered PPP (solid-thick) from approximately 20 degrees ATDC to 10 degrees ATDC. Also shown are the corresponding filtered estimations using the peak-finding algorithm (solid-thin), MLP (dashed-thin) and Gaussian curve fit (dashed-thick). The load is approximately $35 \%$ of MBT on average.

The peak-finding algorithm shows close correspondence to the filtered PPP, except for the last 100 cycles. The MLP has problems finding PPPs below 10 degrees ATDC and is thus biased in those regions.

The second batch is a long ramp in PPP from approximately 10 degrees ATDC to 20 degrees ATDC. The peak-finding algorithm performs well giving unbiased estimations on average. Once more, the MLP has problems in the end regions, showing a significant bias for PPPs later than 18 degrees ATDC. The Gaussian fit algorithm does not perform well and is underestimating the PPP continuously, indicating a significant bias.

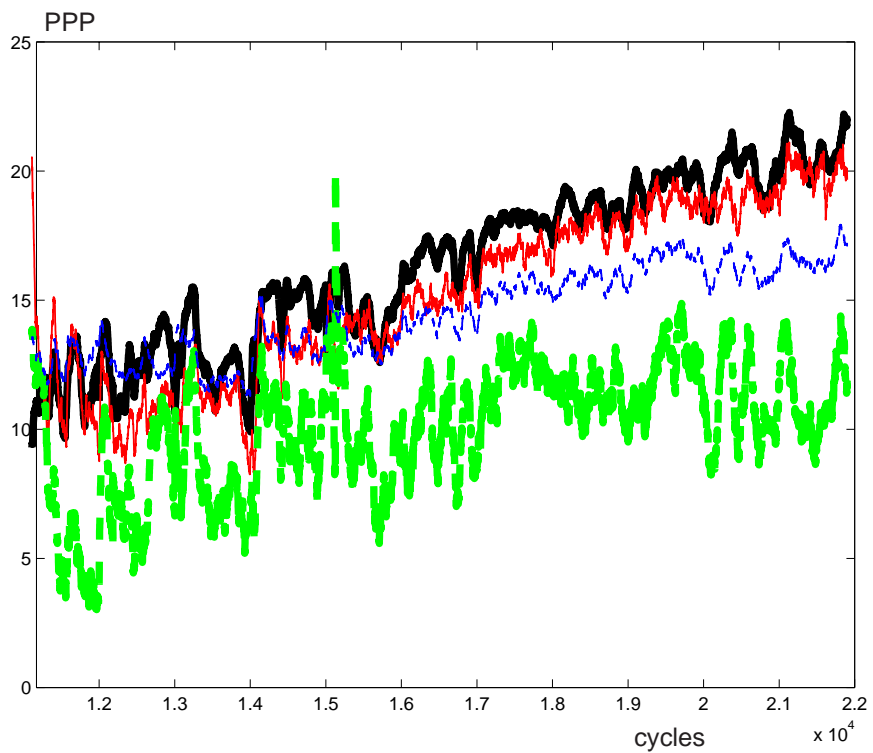

Figure 6. Ramp in low pass filtered PPP (solid-thick) from approximately 10 degrees ATDC to 20 degrees ATDC. Also shown are the corresponding filtered estimations using the peak-finding algorithm (solid-thin), MLP (dashed-thin) and Gaussian curve fit (dashed-thick). The load is approximately $20 \%$ of MBT on average.

The last batch is a close up, illustrating the performance of the algorithms for an oscillating PPP at low load.

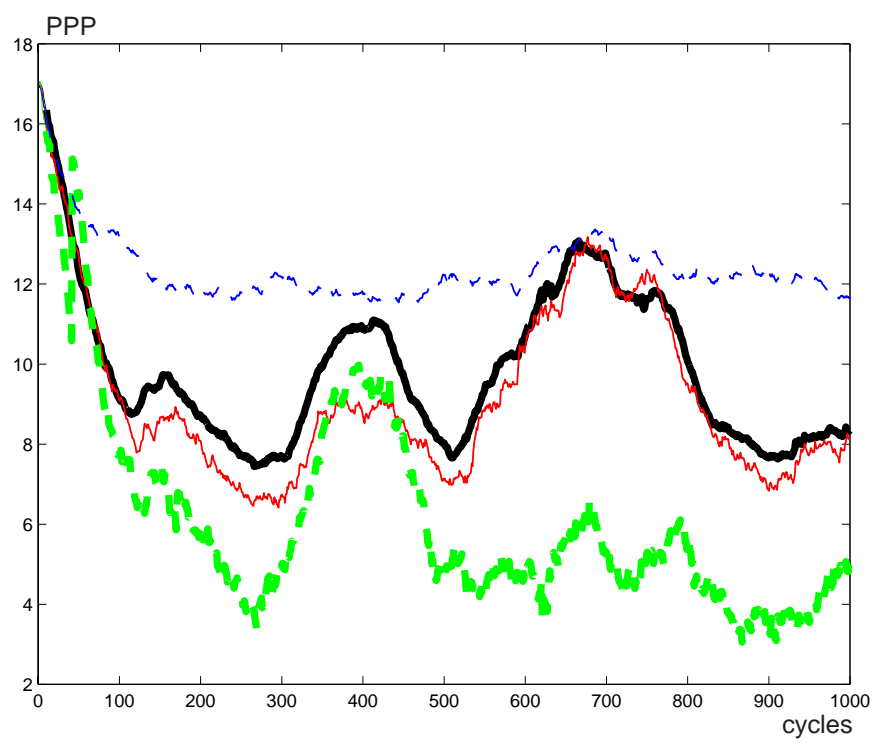

Figure 7. Close up of low pass filtered PPP (solid-thick) and corresponding filtered estimations using the peak-finding algorithm (solid-thin), MLP (dashed-thin) and Gaussian curve fit (dashed-thick). The load is approximately $10 \%$ of MBT on average. 
From the three figures shown here, it is obvious that the MLP has a more narrow range than its competitors. Thus, it has problems estimating small and large PPPs. Furthermore, the Gaussian fit algorithm has, on average, a bias of a few degrees. Moreover, its estimates clearly deteriorate when engine load is low.

\section{CONCLUSION}

A new ion-current based algorithm for on-line estimation of the in-cylinder pressure peak position of spark ignited engines is proposed. The algorithm is based on a simple peak-finding technique utilizing simple least squares fit. Furthermore, the algorithm is confident in the sense that it quantifies the shape of the ion current using a curvature measurement. The peak-finding algorithm outperforms the state of the art models considered here. In particular, the ability of providing unbiased estimates in the most important PPP range is a significant feature. Additionally, robustness towards the low load scenario is demonstrated. Thus, the peak-finding algorithm is the best candidate for PPP feedback to an online controller.

\section{ACKNOWLEDGMENTS}

This work has been supported by the Volvo research foundation, Volvo educational foundation and Dr. Pehr G. Gyllenhammar research foundation.

Ph.D. Thorsteinn S. Rögnvaldsson has provided the results for the Gaussian fit algorithm.

\section{CONTACT}

Magnus.Hellring@ide.hh.se

Ulf.Holmberg@ide.hh.se

\section{REFERENCES}

[1] Glaser, I. and J. D. Powell (1981). Optimal Closed-loop Spark Control of an Automotive Engine. SAE Technical Paper 810058. Society of Automotive Engineers.

[2] Hubbard, M., Dobson, P.D., and Powell, J.D. (1976). Closed Loop Control of Spark Advance using a Cylinder Pressure Sensor. (ASME) Journal of Dynamic Systems, Measurement, and Control. December, pp. 414-420.

[3] Eriksson, L. (1999). Spark Advance Modeling and Control. Ph.D. Thesis, Linköping University. Linköping Studies in Science and Technology No. 580.

[4] Hellring, M., Rögnvaldsson, T. and Wickström N. (2000). Ion Current Based Pressure Peak Detection Under Different Air Humidity Conditions. Advanced Microsystems for Automotive Applications 2000. pp. 125138.

[5] Hellring, M., Munther, M., Rögnvaldsson, T. and Wickström N. (1999). Spark Advance Control using the Ion Current and Neural Soft Sensors. SAE Technical Paper 991162. Society of Automotive Engineers.

[6] Saitzkoff, A. Reinmann, R., Berglind, T. and Glavmo, M (1996). An ionization equilibrium analysis of the spark plug as an ionization sensor. SAE Technical paper 960337, Society of Automotive Engineers.

[7] Saitzkoff, A. Reinmann, R., Mauss, F. and Glavmo, M (1997). In-Cylinder Pressure Measurements Using the Spark Plug as an Ionization Sensor. SAE Technical Paper 970857. Society of Automotive Engineers.

\section{DEFINITIONS, ACRONYMS, ABBREVIATIONS}

\section{MBT:}

Maximum Brake Torque

PPP:

Pressure Peak Position

TDC:

Top Dead Center

ATDC:

After Top Dead Center

MFB50\%:

Mass Fraction Burned equals 50\% 